\title{
CLEAN UP! \\ »HEIMATSCHUTZ«, ANTI-DROGENPOLITIK UND DIE Rave-Kultur nach dem 11. SePtember 2001
}

\author{
Jörg Fachner
}

\section{Einleitung}

Als die Beastie Boys 1986 mit ihrem Song "You Gotta Fight For Your Right To Party« die Charts eroberten, hätte sich niemand vorstellen können, dass dieser Slogan einmal eine politische Forderung für bedrohte Konzertveranstalter und Clubbesitzer werden könnte. Wie Andrian Kreye in seinem Bericht über ein von den Beastie Boys organisiertes Benefiz-Konzert im Oktober 2001 zu Gunsten der Opfer von 9/11 schrieb, »erinnerten sie New York an ihren alten Schlachtruf, der angesichts von Betroffenheitswahn und Patriotenschmalz mehr gilt als je zuvor: >Fight for your right to party! «. Denn in der Jugend- und Popkultur gehört auch das zur modernen Zivilgesellschaft« (Kreye 2001).

Doch mehr noch: Lässt sich eine >gute Party< ohne Drogen feiern? Schon seit Jahrhunderten beantwortet das Oktoberfest in München diese Frage mit >Nein<, und die Verbindung von bayrischer Blasmusik und Droge zieht Touristen aus aller Welt zur großen Intoxikationsfeier. Ethnologen und Kunsthistoriker betrachten die Verbindung aus Festivität, Musik, Tanz und Drogenkonsum in den verschiedenen Kulturen der Welt schon nahezu als anthropologische Konstante (Hutson 2000; Kupfer 1996; Rätsch 1992; Rouget 1985). In den USA ist man seit dem 30. April 2003 der ambitionierten Überzeugung, dass Tanz- und Musikveranstaltungen den Konsum kontrollierter Substanzen begünstigen und etwas dagegen unternommen werden muss. Als Rezipient eines Newsletters der US-amerikanischen Drug Policy Alliance (www.lindesmith.org) bekam ich am 4. März 2003 die erste Info- 
Mail ${ }^{1}$ ( Action Alert<) mit dem Hinweis, dass der US-amerikanische Kongress versuchen würde Live-Musik und Tanzveranstaltungen zu verbieten, weil dort verbotene Substanzen konsumiert werden könnten. Am 11. April passierte das Gesetzesvorhaben den Kongress ohne Anhörung und am 30. April unterschrieb Präsident George W. Bush das Gesetz.

Sind solche Entwicklungen auch Folgen von 9/11 oder sind sie davon unabhängig und Drogenkonsum und Partykultur werden als salte Bekannte attackiert, weil sich so von anderen Zielen ablenken lässt? Seit 9/11 werden Freiheitsrechte aus Gründen des »Heimatschutzes« verstärkt eingeschränkt. Konzertveranstalter und Clubbesitzer, Musiker, DJs und Medienvertreter fürchten nun um ihr Recht auf Veranstaltungen und weitere Einschränkungen.

\section{Kontrolle des Veranstaltungsortes}

Ohne hier genauer auf den Paragraphendschungel und die juristischen Vorgänge von Eingaben zur Gesetzesänderung, deren Status, Erweiterung und Bezugnahme auf andere, bereits bestehende Gesetze in den USA eingehen zu können, lässt sich der Hergang folgendermaßen darstellen: ${ }^{2}$

Die Gesetze, um die es hier geht, sind zumeist nur kurze Sätze mit Definition potentieller Zielgruppen, des Tatbestandes, seiner Bestrafung etc. Doch diese Sätze werden in bereits bestehende (z.B. Controlled Substances Act) oder zu verabschiedende Gesetze (z.B. PROTECT Act, CLEAN-UP Act) eingefügt und können im Zusammenhang mit anderen bestehenden und noch zu verabschiedenden Gesetzestexten ihre Wirkung auf Musik- und Tanzveranstaltungen entwickeln. So hatte Senator Joe Biden am 8. April 2003 den von ihm und anderen Senatoren schon im Juni 2002 formulierten aber abgelehnten - RAVE Act ${ }^{3}$ in leicht veränderter Form in den am 30. April verabschiedeten PROTECT Act (Statute 151) als Abschnitt 608 unter dem Namen Illicit-Drugs-Anti-Proliferation-Act eingefügt (Drug Policy Alliance 2003b).

1 Die Info-Mails können beim Verfasser zur Einsicht angefordert werden. Online sind sie nicht verfügbar.

2 Siehe hierzu http://www.lindesmith.org/library, auch http://www.protectlive music.org (Stand v. 20.1.2004). Die jeweiligen Gesetze und ihr derzeitiger Status lassen sich unter http: //thomas.loc.gov finden.

3 Abkürzung für: Reducing Americans' Vulnerability to Ecstasy Act. 
Der PROTECT Act ${ }^{4}$ ist ein Gesetz zum Schutz vor Kindesmissbrauch. Doch nachdem im Gesetzestext zunächst über verschiedene Formen des Kindesmissbrauchs, Pornographie und Internetdarstellungen beschieden wird, kommt es unter »Verschiedenes« relativ unvermittelt zum Thema:

»(b) OFFENSES-

(1) IN GENERAL- Section 416(a) of the Controlled Substances Act (21 U.S.C. 856(a)) is amended -

(A) in paragraph (1), by striking >open or maintain any place < and inserting sopen, lease, rent, use, or maintain any place, whether permanently or temporarily, $<$; and

(B) by striking paragraph (2) and inserting the following:

(2) manage or control any place, whether permanently or temporarily, either as an owner, lessee, agent, employee, occupant, or mortgagee, and knowingly and intentionally rent, lease, profit from, or make available for use, with or without compensation, the place for the purpose of unlawfully manufacturing, storing, distributing, or using a controlled substance « (»PROTECT Act« 2003).

Im Abschnitt 608 des PROTECT Act geht es in erster Linie darum der Exekutive den juristisch abgesicherten Zugang zu dem Ort zu verschaffen, an welchem die Straftat, nämlich Verbreitung, Gebrauch usw. von kontrollierten Substanzen, geschieht. Von Musik- oder Tanzveranstaltungen ist hier noch nirgendwo die Rede, doch lässt sich durch die Ausweitung der im Controlled Substances Act formulierten Ortsbefugnisse schon erkennen, dass insbesondere durch den neuen Absatz (B) durchaus Musik- und Tanzveranstaltungen betroffen sein können. Zudem findet sich im Abschnitt (g) Folgendes:

»(g) AUTHORIZATION OF APPROPRIATIONS FOR DRUG EDUCATION. There is authorized to be appropriated such sums as necessary to the Drug Enforcement Administration of the Department of Justice to educate youth, parents, and other interested adults about club drugs« (»PROTECT Act« 2003).

Die Erwähnung des Begriffes »club drugs« im Zusammenhang mit Fördermitteln zur Drogenaufklärung Jugendlicher ist der zunächst einzige Bezug zu dem von der Drug Policy Alliance befürchteten Verbot von Tanz- und Musikveranstaltungen. Wie Senator Biden in seiner Begründung zur (gescheiterten) Einführung des RAVE Act von 2002 ausführte, sind »club drugs « Bestandteil der Rave-Kultur:

4 Abkürzung für: Prosecutorial Remedies and $\underline{\text { Other }}$ Tools to end the Exploitation

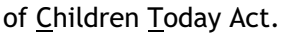


»(3) The trafficking and use of >club drugs‘, including 3, 4-Methylenedioxymethamphetamine (Ecstasy or MDMA), Ketaminehydrochloride (Ketamine), Flunitrazepam (Rohypnol), and Gammahydroxybutyrate (GHB), is deeply embedded in the rave culture $«(» R A V E$ Act $« 2002)$.

Es blieb nicht allein bei Senator Bidens Vorstoß. Andere Gesetzestexte liegen zur Verabschiedung vor, welche gezielter gegen Clubs und Veranstalter angewandt werden können. Auch wenn das allgemeine Strafmaß für Verbreitung, Herstellung, Besitz usw. von kontrollierten Substanzen und besonders von »club drugs « schon in Sektion 608 des PROTECT Act 250.000 Dollar oder das Doppelte des durch die kontrollierten Substanzen erwirtschafteten Gewinns beträgt (»PROTECT Act« 2003), wendet sich die Sektion 305 des CLEAN-UP Act dann explizit an »Promoters of commercial drug-orientated entertainment «. Die Sektion 305 des CLEAN-UP Act wurde - wie schon beim PROTECT Act - als ein kurzer Einschub in ein Gesetz formuliert, welches in der Hauptsache der Definition und Verfolgung illegaler Amphetamin-Labors dient. Folgendes findet sich dann aber im Abschnitt »Enforcement«:

»SEC. 305. LIABILITY OF PROMOTERS OF COMMERCIAL DRUGORIENTED ENTERTAINMENT.

(a) IN GENERAL. - The Controlled Substances Act is amended by inserting after section 416 (21 U.S.C. 856) the following new section:

SEC. 416A. PROMOTERS OF COMMERCIAL DRUG-ORIENTED ENTERTAINMENT.

,Whoever, for a commercial purpose, knowingly promotes any rave, dance, music, or other entertainment event, that takes place under circumstances where the promoter knows or reasonably ought to know that a controlled substance will be used or distributed in violation of Federal law or the law of the place where the event is held, shall be fined under title 18, United States Code, or imprisoned for not more than 9 years, or both « (»CLEAN-UP Act « 2003).

Hier wird deutlich ein Zusammenhang von Musikstil, Veranstaltungsform und Drogenkonsum hergestellt.

Der CLEAN-UP Act ${ }^{5}$ wendet sich hauptsächlich gegen illegale Labors, in denen Methamphetamine wie Ecstasy und Speed - aber vielleicht ja auch Anthrax und ähnliches - hergestellt werden. Nach 9/11 und den anschlieBenden Anthraxanschlägen entstand der Verdacht, dass solche Labors möglicherweise auch Terroristen zur Verfügung stehen könnten und des Weiteren Überschüsse aus Rave-Parties zur Finanzierung terroristischer Aktivitäten dienen könnten. Dies führte zu der Formulierung des »Justice

5 Abkürzung für: $\underline{C l e a n, ~ L e a r n, ~ E}$ Educate, $\underline{A}$ bolish, Neutralize, and $\underline{\text { Undermine }}$ Production Act. 
Enhancement and Domestic Security Act « - im Juristenlatein $\gg S$. 22 abgekürzt und vorgeschlagen von Senator Tom Daschle (»Justice Enhancement and Domestic Security Act of 2003« 2003). Dieses Gesetzesvorhaben versucht, das seit zwanzig Jahren gültige >Crackhouse Law <m Passagen des RAVE Act zu erweitern. Effektivität und Nutzen eines solchen Gesetzes zur Terrorismusbekämpfung beurteilt Dave Kopel in seinem Artikel über Daschles Gesetzesvorhaben schon aufgrund der Kompetenzverschiebung von lokalen und nationalen Autoritäten folgendermaßen:

»The idea is to make the promoters of musical events liable for drug consumption at those events - even when the consumption is entirely incidental, and has nothing to do with any action by the promoters. The legislation is, in effect, an admission of failure by the Drug Enforcement Agency: Unable to control drug use, it's looking to force concert promoters and theaters to do it, on pain of imprisonment. [...]

Under a federal system, it is supposed to be state and local governments, and not the federal government, that deal with matters of local law enforcement. There's nothing in the Constitution authorizing the federal government to waste taxpayer money seducating ( the public about the dangers of swing music, rave music, or any other form of music.

We are continually reminded, as a justification for restrictions on civil liberties, that we are at war. But, precisely because we are at war, we cannot afford to have the Justice Department and federal law-enforcement authorities wasting their time on the kind of trivia represented by much of S. 22, including the misnamed >crackhouse< provision. The Constitution gives Congress and the federal government the duty to provide for the national defense; to allow them to exercise never-granted powers over local crimes and music styles is to undermine that essential focus on national defense« (Kopel 2003).

Auch wenn Veranstalter, Club- oder Hallenbesitzer selber alle Möglichkeiten nutzen um zu verhindern, dass keiner der Gäste etwas Verbotenes konsumiert, reichen diverse Verdachtsmomente, die unten näher erläutert werden, reicht eine einzige ertappte Person aus, das Gesetz in Kraft treten zu lassen. Allein die Tatsache, dass sie ihren Helfern etwas zum Thema »Erste Hilfe « und »Safer Use « beibringen oder für Ambulanz und medizinische Hilfe bei großen Tanzveranstaltungen gesorgt haben, kann den Veranstaltern Schwierigkeiten einbringen (Drug Policy Alliance 2003a). In den USA muss dann jeder Club- oder Hallen-Besitzer, jeder Veranstalter eines Konzertes, Festivals oder einer Rave-Party dafür Sorge tragen, dass auf seiner Veranstaltung kein Besucher eine verbotene Substanz konsumiert. Geschieht es dennoch und wird von einem anwesenden Gesetzeshüter bemerkt, kann die Veranstaltung sofort verboten werden und der Veranstalter muss mit einer 
Strafe von bis zu 250.000 Dollar oder einer Gefängnisstrafe von bis zu neun Jahren rechnen.

Am 25. Juli 2003 wurde von vier weiteren Senatoren der Ecstasy Awareness Act beantragt (»Ecstasy Awareness Act « 2003). Sie verlangten u.a. eine Verschärfung des Strafmaßes und beantragten, das höchste Strafmaß solle 500.000 Dollar oder 20 Jahre Gefängnis betragen, wenn der Veranstalter hätte wissen können oder sollen, dass auf seiner Veranstaltung verbotene Substanzen konsumiert werden (Drug Policy Alliance 2003b). Doch nicht nur professionelle Veranstalter sind im Visier. Auch wenn jemand zu Hause eine private Party feiert, bei der ein Konsum illegaler Substanzen nachgewiesen werden kann, muss der Gastgeber mit einer harten Geld- oder langen Gefängnisstrafe rechnen.

Einen Monat nach Inkrafttreten des PROTECT Act wurde die Sektion 608 im Mai 2003 in Billings, Montana, erstmalig angewandt. Die Betroffenen waren die Organisationen NORML (National Organisation for the Reform of Marihuana Laws) und "Students for a sensible Drug Policy«, die eine gemeinsame Benefizveranstaltung für ihre Projekte geplant hatten. Da es bei den hier diskutierten Gesetzen für die Exekutive darum geht, eine rechtlich abgesicherte Kontrolle über die Veranstaltungsorte zu bekommen, wurden den Betreibern des Veranstaltungsortes »Eagle Lodge« mit 250.000 Dollar Strafe gedroht, falls bei einem der Besucher der Veranstaltung Marihuana gefunden würde. Die Betreiber sagten die Veranstaltung ab (hierzu Sullum 2003).

Weitere Anwendungen der Gesetze belegt William D. McCull in Florida, Wisconsin und New Orleans: ${ }^{6}$

»There have already been prosecutions of owners and promoters of nightclubs in which there were no allegations that the defendants themselves were involved in any drug activity. Even clubs with strong anti-drug policies have been targeted. In Panama City, Florida, prosecutors went after a nightclub that publicized and enforced zero-tolerance drug policies, had well-trained security, and consistently reported drug-related incidents to local police. In New Orleans, a certain club took these same precautions and even went so far as to have its security staff hold patrons caught in possession of drugs while the police were on route.

Even the patrons themselves have become the victims of overzealous policing. In Wisconsin, local officials recently raided a popular nightclub and tried to give $\$ 1000$ fines to 442 customers. With help from the ACLU, most of the clubgoers were able to have their charges dismissed. A total of only three customers were charged with actual drug violations« (McColl 2003).

6 Siehe hierzu auch http://www.emdef.org/laws_and_cases.html. 


\section{Jugendschutz}

Eine Reaktion auf den 11. September 2001 war die Einrichtung eines Ministeriums für Heimatschutz (DHS = Department of Homeland Security), dessen mittlerweile 18.000 Mitarbeiter zahlreiche der in der Verfassung vormals verbürgten demokratischen Grundrechte je nach Bedarf außer Kraft setzen können, um die unsichtbare Gefahr des Terrorismus zu bannen (hierzu Drug Policy Alliance 2003c). Damit tritt das Amerika der Gegenwart eine Reise in die dunkle Vergangenheit der gerade in Deutschland gut bekannten Gesinnungsüberprüfung an. Die Konsequenzen der in den Gesetzesvorhaben formulierten Straftatbestände sind drakonisch und bedrohen die freie musikalische und sprachliche Meinungsfreiheit. So sehen dies kritisch zur US-Drogenpolitik eingestellte Verbände wie z.B. die von der George Soros Foundation unterstützte Drug Policy Foundation (Drug Policy Alliance 2003a; 2003b; McColl 2003), die American Civil Liberties Union (http://www. aclu.org), aber auch MDMA-Experten wie Marsha Rosenbaum (2002) oder Vertreter von Unterhaltungsindustrie und Musikerverbänden wie z.B. der Electronic Music Defense and Education Fund (http://www.emdef.org; Kopel 2003; Sokoni 2004; Sullum 2002).

Durch zahlreiche Proteste von in der Tanz-, Musik- und Unterhaltungskultur involvierten US-Organisationen ${ }^{7}$ konnte der ursprüngliche RAVE Act entschärft werden: Die von Senator Biden bei Vorlage vor dem Senat am 18. Juni 2002 benannten Indizien für Drogengebrauch wie der Verkauf von Wasser, die Gegenwart von bunten Glowsticks oder von Massageöl (vgl. »RAVE Act « 2002) wurden aus der Indikatorenliste gestrichen. Ebenso ist es weiterhin möglich eine Rave-Party zu bewerben, ohne dass schon die bloße Nutzung des Wortes »Rave« das Gesetz in Kraft treten lässt.

»Ignoring the efforts made by these clubs, prosecutors instead claimed that the sale of bottled water and an onsite ambulance showed that owners knew about and encouraged drug use. Prosecutors presented glow sticks, blow pops, and pacifiers (all legal items) seized from the venues as evidence that patrons were using drugs. In the Florida case, prosecutors showed jurors a video of a man massaging another man's back, stating that somehow this was evidence of drug use. (It leads us to question whether or not the prosecutors were attempting to sway the jury through blatant homophobia.) Fortunately, the jury didn't buy these ridiculous cases« (McColl 2003).

7 Siehe hierzu http://www.emdef.org; http://www.roargroup.org; http://www. liquidsoundlounge.com; http://www.legalizedancingnyc.com; http://www. protectlivemusic.org oder http://www.dancesafe.org (Stand v. 26.4.2004). 
Senator Bidens Motto zur Begründung des RAVE Act von 2002 war offensichtlich: »Veranstalter machen Kasse auf Kosten unserer Kinder «, entsprechend war seine Argumentation in der Senatsanhörung vom 18. Juni 2002 aufgebaut (vgl. http://www.emdef.org/s2633). Gegen solche Ausbeutung von Kindern und Jugendlichen sollten RAVE Act und CLEAN-UP Act demnach schützen. Wasser würde absichtlich zu teuer verkauft, um die von Tanz und Droge schwitzenden und entsprechend dehydrierten Raver abzukassieren. Biden argumentierte bei der ersten Eingabe damit, dass Veranstalter mit dem Etikett der >alkoholfreien Parties - den Eltern vorgaukelten, ihre Kinder seien sicher. Dass es sich bei dem Begriff Ecstasy aber nicht allein um die Tanzekstase handele, sondern um die Droge MDMA, würde verschwiegen. Außerdem seien die Sicherheitskräfte der Veranstalter nur zum Schein angestellt (vgl. »RAVE Act « 2002). ${ }^{8}$

»Last spring, the Chicago City Council decided to scrack down on wild rave parties that lure youngsters into environments loaded with dangerous club drugs, underage drinking and sometimes predatory sexual behavior, $<$ as the Chicago Tribune put it. The newspaper described raves as >one-night-only parties ... often held in warehouses or secret locations where people pay to dance, do drugs, play loud music, and engage in random sex acts.<

In the case of sclub drug, adolescents are both victims and perpetrators. Parents fear for their children, but they also fear them. When Mayor Daley warned that sthey are after all of our children, $<$ he may have been imagining predators in the mold of Fu Manchu or Charles Manson. But the reality is that raves - which grew out of the British racid house< movement, itself reminiscent of the psychedelic dance scene that emerged in San Francisco during the late '60s - are overwhelmingly a youth phenomenon« (Sullum 2002).

Angst und Sorge der Eltern vor potentiellen Gefahren der Jugendzeit werden offensichtlich von den Initiatoren solcher Gesetze instrumentalisiert, um ein sich tradierendes Jugendphänomen, die Veranstaltung von Parties, um die hier und dort möglicherweise extrem ausgelebten Übergänge vom Kind zum Erwachsenen kontrollieren zu können. In solchen Entwicklungsphasen kann je nach Person ein Interesse an veränderten Bewusstseinszuständen und Drogen entstehen. Dass Jugendliche mit Gleichaltrigen (auf Parties) tanzen, mit dort möglicherweise erworbenen Bekanntschaften sexuelle Erfahrungen machen und Emotionen erleben wollen, erscheint ebenso als eine anthropologische Konstante wie die eingangs erwähnte Beobachtung, dass zu festlichen Anlässen und zu Übergangs- und Initiationsritualen seit Menschheitsbeginn emotionale und sinnliche Intensitätsverstärker in Form von Drogen

8 Hintergründe, Gesetzestext und Protokoll dieser Senatssitzung vom 18. Juni 2002 finden sich unter http://www.emdef.org/s2633. 
konsumiert wurden. Dass Rave-Parties, ihre 'Location das lange Tanzen, Ecstasy-Konsum und Übernächtigung zudem ihren Teil zu außergewöhnlichen Erfahrungen und Bewusstseinszuständen beisteuern, ethnographierte Scott R. Hutson (2000) anhand von Rave-Diskussionsforen im Internet.

\section{Marihuana Tax Act und »moralische Unternehmer«}

Mit der Sorge der Eltern um ihre Kinder wurde auch schon zu Beginn des letzten Jahrhunderts Druck auf die Gesetzgeber ausgeübt. Schon in den 1930er Jahren schaffte es der Leiter des FBN (Federal Bureau of Narcotics), Harry Anslinger, den MARIHUANA-TAX-Act durchzubringen. Das Strickmuster war dem des RAVE Act ziemlich ähnlich. Auch schon damals wurden Tanzveranstaltungen und Konzerte populärer Musik als Urheber des Drogenelends und der schlechten Leistungen von Studenten, Schülern, Angestellten und Arbeitern des Landes benannt. Dies geschah durch eine Verbindung von Desinformation und Beschuldigungen der zumeist schwarzen Jazzmusiker als Drogenpropheten. "We had more jazz bands in jail in the 1930s than I can count«, sagte Anslinger in einem Gespräch mit dem Drogenhistoriker David Musto (1997).

Der Soziologe Howard S. Becker beschreibt die Kriminalisierung von Marihuana in den USA als ein Produkt »moralischen Unternehmertums «. Menschen, die eine Initiative ergreifen, um das von ihnen als abweichend definierte Verhalten anderer sozialer Gruppierungen und Altersgruppen zu stigmatisieren, nennt er »soziale Unternehmer (Becker 1973: 121-148).

Rassistische, politische und kulturelle Motive wurden damals zurechtgebogen und in der von Patrick Hearst begründeten >Yellow Press - dem uninformierten Bürger ein Cocktail von Gewalt, Verbrechen und Zügellosigkeit aufgetischt. Folgender Zeitschriftenausriss aus den 1930er Jahren verdeutlicht die geschürte Sorge um die Kinder: 


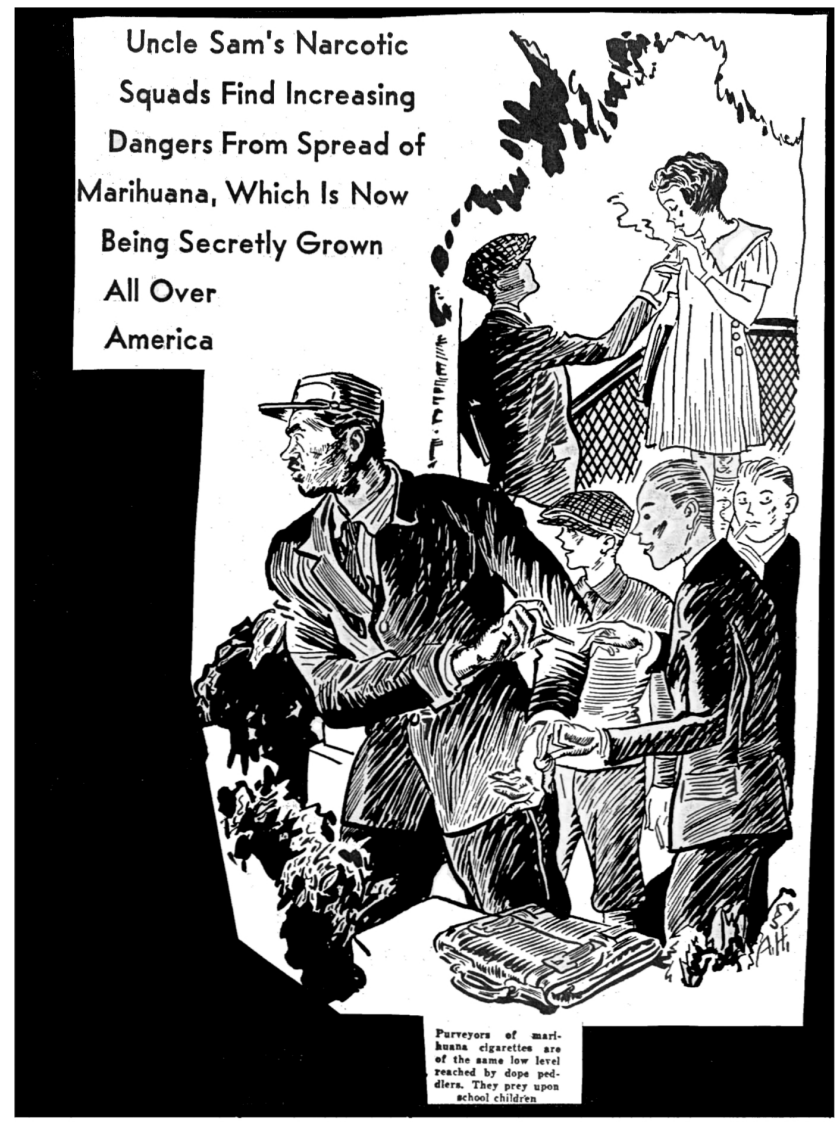

»Lieferanten von Marihuana-Zigaretten befinden sich auf dem gleichen niederen Niveau wie Drogenhändler. Sie rauben Schulkinder aus." (Ausriss aus einer nicht identifizierten Zeitschrift der 1930er Jahre in der Harry J. Anslinger Collection, Box 9, Pennsylvania State University, Dept. of Rare Books and Historic Archives).

\section{Reefer Madness, Tanzlokale und Jazz}

In dem Film Reefer Madness, der 1936 zu Aufklärungszwecken hergestellt wurde, wird eine Situation konstruiert, in welcher junge Erwachsene sich zum Swingtanzen verabreden und in der ein Musiker am Klavier den besonderen > Jive< dafür hat, weil er sich ab und an in ein Hinterzimmer zurückzieht und Marihuanajoints raucht (hierzu Shapiro 1989: 52ff.; Sloman 1998). 
Es wird suggeriert, dass die 'wilde Rhythmik und >chaotische Struktur der Jazzmusik einem ausschweifenden und unmoralischen Lebensstil entsprängen, die geistige Wurzel dieser hedonistisch, musikalischen Verirrungen im Cannabisrausch zu suchen sei und dass »diese satanische Musik und der Genuß von Marihuana weiße Frauen dazu brächten, ssexuelle Beziehungen mit Negern zu wollen« (Herer 1993: 150). Neil Leonard zitiert in seinem Buch Jazz and the White Americans den Orchesterleiter des State Hospital in Napa, Kalifornien, der 1923 folgendes Statement machte:

"I can say from my own knowledge that about fifty per cent of our young boys and girls from the age of 16 to 25 that land in the insane asylum these days are jazz-crasy dope fiends and public dance hall patrons. Jazz combinations - dope fiends and public dance halls - are all the same, >one $<$. Where you will find one you will find the other « (zit. n. Leonard 1962: 37).

Tanzlokale waren auch schon dem Ölmagnaten John David Rockefeller ein Dorn im Auge, trafen sich dort doch nach seinen Erkenntnissen Arbeiter, um Gewerkschaften zu gründen. Als >moralischer Unternehmer f finanzierte er schon während der US-amerikanischen Alkoholprohibition die >Anti-Saloon League`, die für die Schließung aller Kneipen eintrat (vgl. Behr 1982: 193). Da Jazz seinerzeit hauptsächlich als Tanzmusik gespielt wurde, kamen Jazzbands als vorgebliche Propagandisten und Hauptvertreiber von Marihuana ins Visier. In der Zeitung von St. Louis fand sich am 8. April 1934 folgender Bericht:

"Alles deutet darauf hin, daß die Droge [Marihuana] von reisenden Jazzbands, die auf Universitäts- oder Studentenverbindungs-Tanzveranstaltungen spielen, in die Universität eingeführt wurde« (zit. n. Shapiro 1989: 61-62).

Anslinger ließ von nahezu jedem bekannten Jazzer in einer speziellen Musikerkartei ein Dossier anlegen, welches Hinweise auf Drogenkonsum dokumentierte (Herer 1993: 150ff.; Shapiro 1989: 61ff.). Diese Dossiers befinden sich heute im Anslinger Archive der Universitätsbibliothek des State College in Pennsylvania. Hier ein Beispiel eines solchen Berichtes, angefertigt durch einen von Anslingers Informanten ${ }^{9}$ :

»These three Negros had arrived three days previously from New York to fill an eight-weeks dancing contract at the Frolics Cabaret, and one of them at least had immediately started in trafficking. [...] Banks and Burnham admitted using marihuana cigarettes and claimed to secure their supply from a tobacco shop in New York City, but refused to give any further details. They

9 Das Original befindet sich in der Pattee Library of Pennsylvania State University, State College, PA. (siehe http://www.libraries.psu.edu/tas/ cataloging/rarebook/541.htm; Stand v. 26.1.2004). 
arrived in Montreal by motorbus direct from New York City, and I understand have for some months past been filling dancing contracts in the United States« (Anslinger 1933).

\section{Stigmatisierung und Informationspolitik}

Jack Herer (1993) beschreibt die Geschichte des Hanfverbotes als die Geschichte einer industriepolitischen Verschwörung. Als Mittel zum Zweck diente die politische Instrumentalisierung von Vorurteilen gegenüber dem Lebensstil von Menschen anderer Hautfarbe. Herers (1993: 146ff.) Ausführungen folgend, war bezogen auf die USA das Cannabisrauchen Anfang des 20. Jahrhunderts hauptsächlich in gesellschaftlich unterprivilegierten Gruppen Usus, also bei den meist in den Armenvierteln der Städte lebenden »Niggern, Mexikanern und Unterhaltungskünstlern«, wie Anslinger 1937 dem US-Kongress mitteilte (zit. n. Herer 1993: 150). Infolge der Weltwirtschaftskrise 1929 wurden insbesondere die mexikanischen Immigranten zu Konkurrenten der US-amerikanischen Arbeitnehmer und sollten nach Mexiko zurückgedrängt werden.

David F. Musto (1999) sieht in der Stigmatisierung von Marihuana und Jazzmusikern den »verzweifelten« Versuch eines mittellosen Beamten, durch gezielte Beeinflussung der Medien ein Verbot zu erreichen. Marihuana sei schließlich 1937 durch einen Steuertrick aus dem Handel gezogen worden, eine Strategie, die langfristig die Drogenpolitik der USA unglaubwürdig und zudem die staatsverdrossenen Hippies der 1960er auf solcherlei verbotene Substanzen neugierig gemacht habe (vgl. Musto 1997). Zweifel an der Lauterkeit staatlicher Drogeninformationspolitik werden auch in neuerer Zeit insbesondere durch TV-Spots verstärkt, in denen Gehirne von EcstasyUsern gezeigt werden (vgl. http: //www.clubdrugs.org), die durch den möglicherweise neurotoxischen Gebrauch >Löcher aufweisen. Solche Spots werden freilich von Jugendlichen nur milde belächelt, wie Rosenbaum (2002: 141) in einer Analyse darstellt.

Marihuana stand in den 1930er Jahren als ein Symbol für die gefürchteten gesellschaftlichen Gruppen, die es durch gezielte Propaganda zu diskreditieren galt (Musto 1997). Dass sich an solcher Vorgehensweise bis heute anscheinend nur die Droge und die Musik geändert haben, verdeutlicht Jacob Sullum anhand eines Zitats von Jonathan Keane:

") The sensationalist reaction that greets every mention of the word Ecstasy in this country is part of a wider, almost unconscious fear of young people, writes Jonathan Keane in the British New Statesman, and the observation 
applies equally to the United States. For >middle-aged and middle-class opinion leaders ... E is a symbol of a youth culture they don't understand « (zit. n. Sullum 2002).

Die Assoziationsreihe von Jazzkeller, Opiumhöhle und Rotlichtmilieu gab und gibt seit Storyville - dem legendären 1917 geschlossenen Hafen- und Vergnügungsviertel von New Orleans - eine vorzügliche Projektionsfläche für Moralisten und Politiker auf der Suche nach einem Feindbild ab. Vor dem -Untergang des Abendlandes< wurde gewarnt, denn die »Reefer Madness « würde durch das »Mörderkraut « die Jugend des Landes im kollektiven Rauschzustand dahinraffen, wie Pater Josef Devine 1943 in seinem Buch Marihuana - Assassin of Youth abermals warnte. Diesen latenten sinneren Feind des Landes galt es zu identifizieren, seine Machenschaften zu benennen und zu tilgen (vgl. Shapiro 1989: 24ff.).

Richard Giulianotti zeigt in seiner Analyse der Darstellung von Drogen in den Medien, wie bis heute bereits stigmatisierte soziale Randgruppen und Personen genutzt werden, um ihnen subkulturelle und als unmoralisch bezeichnete Tendenzen und Aktivitäten zu unterstellen, welche dämonenhaft latent unter der Oberfläche auf ihren Durchbruch und auf öffentliche Aktivität warten (vgl. Giulianotti 1997: 425ff.). Cannabis-Konsum und Jazzmusik wurden in der Vergangenheit zu asozialen Machenschaften erklärt, die alsbald die Gesellschaft ruinieren würden. Rave-Parties und Ecstasy-Konsum scheinen das heutige Äquivalent zu sein (vgl. Rosenbaum 2002).

\section{Chill-Out - Sozialpharmakologische und soziophysiologische Aspekte von Drogen und Partykultur}

Ähnliche Hetzkampagnen galten Anfang des 20. Jahrhunderts den Chinesen und ihren »Opiumhöhlen«. Wie die Mexikaner und Schwarzen wurden Chinesen als billige Arbeitskräfte und Menschen zweiter Klasse behandelt. Ihre kulturellen Traditionen waren zudem unerwünscht. Shapiro beschreibt, wie ein Teil chinesischer Kultur - der Genuss von Opium in einem Setting mit bequemen Liegen, gedämpftem Licht und leiser Musik - zu einer weiteren Ausgrenzungsschablone seitens der Cannabis-Prohibitionisten wurde.

»Die bessere Gesellschaft hielt sich natürlich von den Chinesen fern, aber die weiße Unter- und die künstlerische Halbwelt hatten keine Skrupel, Beziehungen zu dieser Gesellschaftsschicht zu pflegen. Im Gegensatz zur einsamen Be- 
schäftigung, die das Morphiumspritzen darstellte, handelte es sich beim Opiumrauchen um eine sehr soziale Angewohnheit, und die Attraktion der leicht zugänglichen Opiumhöhle war für Chinesen und Weiße gleich faszinierend. Zubehör und Ritual des Opiumrauchens, das Bewusstsein, dazuzugehören und Teil einer exklusiven Schicht zu sein, das die Raucher hatten, die Verwendung eines geradezu esoterischen Jargons - shop joint< (Drogenlokal), >long draw` (ein tiefer Zug an der Pfeife), 'yen` (Gier, Abhängigkeit) usw. - , das alles sollte zu einem Muster werden, dem auch die Drogensubkulturen des darauf folgenden Jahrhunderts folgen würden« (Shapiro 1989: 26).

Dass Drogenwirkungen durch das Zusammenwirken von Set (physiologische und psychologische Konstitution) und Setting (soziale und physikalische Umgebung) beeinflusst und - was an sinnlichen Reizungen durch Drogen und Kontext erlebt und interpretiert wird - gestaltet werden können, ist eine in den Drogenkulturen und -therapien bekannte Tradition (Leary 1997; Weil 1998; Zinberg 1984). Durch das Wirkungsprofil der Drogen bildet sich eine soziophysiologisch vorübergehend ähnlich verlaufende Wirkung und entsprechende Bevorzugung von äußeren Reizkombinationen heraus. Jill Jonnes (1999: 119ff.) schildert, wie in den Harlem Tea-Pads (Teestuben) und Jazzkellern im New York der 1930er Jahre ein (auch im heutigen Chill-OutBereich von Rave-Parties durchaus übliches) >Lounge-Setting aus Teppichböden, gedämpftem blauen Licht, Sesseln usw. entstand. Marihuana konsumierende Musiker und Tänzer im New York der 1930er Jahre bevorzugten ein Setting, wie es ein Zeitungsartikel von 1936 beschreibt:

»Early in the morning, when night club singers, musicians and dancers are through work, they gather informally - these affairs apparently are never arranged - and have a few drinks. With their uncanny power for wheedling melody out of even the worst pianos, it isn't long before the crowd is humming, softly clapping hands or dancing in sensuous rhythms that have never been seen in nightclubs. There is little noise; windows are shut, keeping the smell of smoking weeds away from what might be curious nostrils. Nor there is any of the yelling, dashing about, playing of crude jokes or physical violence that often accompany alcoholic parties; under the influence of marihuana, one has a dread of these things « (Anon. 1936: 8).

Es ergibt sich die Frage, ob solche sozialpharmakologischen Aspekte von Set und Setting (man denke nur an die von Senator Biden als Indizien von Ecstasy-Konsum diskutierten Glowsticks) als ein Teil der Drogenkultur oder als ein soziophysiologisch gemeinsam geteilter und bevorzugter Bereich für Erholung, Entspannung und Konversation auf Tanzveranstaltungen betrachtet werden soll? Als ein Ort, an dem Menschen sich setzen oder entspannt 
hinlegen um zu regenerieren oder nachzudenken, zu plaudern oder vor sich hin zu träumen. So findet sich in dem von Shapiro beschriebenen Muster auch die oben dargestellte, scheinbar an den Haaren herbei gezogene Begründung der Indizien für Drogengebrauch (Glowsticks, Massageöl usw.) wieder. Doch lässt sich eine aus dem Blickwinkel der Drogenverfolgung gebildete Indizienkette natürlich nicht für alle Besucher einer Rave-Party oder einer Tanzveranstaltung im Jahr 1936 süber einen Kamm scheren<, wie Vertreter von Bürgerrechtsverbänden betonen. Das musste auch Richter Thomas Porteous aus New Orleans anerkennen: "the government cannot ban inherently legal objects [...] because a few people use the same legal items to enhance the effects of an illegal substance (zit. n. Rosenbaum 2002: 141). Auch wenn manche scheinbar durch Drogenreizungen Freude an solchen Umgebungsfaktoren entwickeln, können sich andere auch ohne Drogen daran erfreuen.

\section{Rave of the brave...}

"Sicherlich ist Marihuana eher harmlos [...], aber die Sache war ein Beispiel dafür, dass ein Verbot die Autorität des Staates stärkt«, sagte Harry J. Anslinger in einem Interview in den 1970er Jahren (zit. n. Behr 1982: 215). Zu seinem großen Bedauern konnte Anslinger sein Ziel nicht durchsetzen: alles, was in der Jazzmusik Rang und Namen hatte - von Louis Armstrong, Dizzy Gillespie, Count Basie bis Lionel Hampton -, in einer Nacht zu verhaften und als Drogenpropheten zu brandmarken. Warum? Sein Vorhaben wurde nach dem Eintritt der USA in den zweiten Weltkrieg vom Verteidigungsministerium abgeblockt, da den Gls an der Westfront in Europa gerade diese Drogen-Jazzmusik die Kampfmoral erhielt (vgl. Sloman 1998: 133-157).

Schlussendlich kam im Sommer 2003 nach einem Fernsehbericht des NDR heraus, dass Flieger der US-Bomber im ersten und zweiten Golfkrieg, in Afghanistan und im Kosovo unter ziemlich hohen Methamphetamindosierungen ihre Kampfeinsätze flogen. Methamphetamin ist Bestandteil der vom RAVE Act tangierten (MDMA) Ecstasy-Pillen. Die Piloten bekamen die Drogen, um wach bleiben zu können, und wenn sie des Öfteren mal das Ziel verfehlten oder falsch interpretierten und dabei einen Konvoi von flüchtenden Zivilisten für Feindbewegungen hielten bzw. Kameraden unter »friendly fire « setzten, war die offizielle Lesart, dass solche Kollateralschäden im Krieg nun mal leider nicht zu vermeiden seien. Hunderte von Fällen kann Marc Herold, Professor an der New Hampshire University, aufzählen, in denen 
Menschen unter Drogeneinfluss getötet wurden - jetzt aber im Auftrag der Regierung (vgl. Doran 2003).

Verbindungen der sich um den RAVE Act herum gruppierenden Gesetzesänderungen zu den Ereignissen des 11. September lassen sich nicht eindeutig aufweisen, aber die Tendenz ist offensichtlich. Gültiges Gesetz ist zum Zeitpunkt der Abfassung dieses Artikels (April 2004) nur die Sektion 608 des PROTECT Act. Doch wer auf dem Server des US-Kongress (http://thomas. loc.gov) nach den in diesem Beitrag angesprochenen Gesetzestexten und -initiativen, nach ihrem gegenwärtigen Status und den Mitschriften der Kongress- und Senatsdebatten sucht, wird feststellen, dass alle diese Gesetzesvorhaben dem subcommittee for Crime, Terrorism and Homeland Security v vorgelegt werden müssen. Die von Senator Daschle eingebrachte Gesetzesinitiative weist hier inhaltlich den deutlichsten Bezug auf das nach den Anschlägen von 9/11 veränderte soziale Klima auf. Die oben beschriebenen Gesetzesinitiativen zielen darauf ab, Veranstalter von Rave-Parties einzuschüchtern, und bedrohen die sprachliche und musikalische Meinungsäußerung. Die noch zur Verabschiedung vorliegenden Gesetzesinitiativen im Kongress und Senat der USA sind Bestandteile einer moralisch unterfütterten Konstruktion, die davon auszugehen scheint, dass die bei kommerziellen Rave-Parties auf Kosten der US-amerikanischen Jugend erwirtschafteten Gewinne in den Terrorismus fließen. So wurde in TV-Spots unterstellt, dass jeder Drogenkonsument den Terrorismus unterstütze (vgl. Drug Policy Alliance 2003c). Auch wenn offensichtlich ist, dass Drogengewinne letztlich dann am höchsten sind, wenn es ein Drogenverbot gibt - da sich durch ein Verbot ja nicht zwangsläufig die Nachfrage ändert, im Gegenteil nur die Preise steigen und die Qualität sinkt (vgl. Behr 1985) -, ändert dieses Wissen um Vorgänge auf dem schwarzen Markt nicht die politische Herangehensweise. "Afghanistan's Taliban profited from the opium trade because of drug prohibition, not in spite of it « (Drug Policy Alliance 2003c).

Was mir bei meinen Web-Recherchen auffiel, war eine häufige Referenz des Beastie Boys-Titels "You Gotta Fight For Your Right To Party« (vgl. bspw. Pollack 2003). Ist das verwunderlich? 


\section{Literatur}

Anon. (1936). »Topics of the Day: Facts and Fancies about Marihuana. «In: The Literary Digest, 24. Oktober, S. 7-8 [Teilfaksimile in: http://users.lycaeum.org/ sputnik/Ludlow/Texts/literary.html; Stand v. 15.6.2004).

Anslinger, Harry (1933). Marihuana Users: Musicians 1933-1937 (Historical Collections and Labor Archives: Harry J. Anslinger Collection, Box 9, File 54). State College, PA.: Pattee Library of Pennsylvania State University.

Becker, Howard S. (1973). Außenseiter. Zur Soziologie abweichenden Verhaltens. Frankfurt/M.: Fischer.

Behr, Hans Georg (1982). Von Hanf ist die Rede. Basel: Sphinx Verlag.

Behr, Hans Georg (1985). Weltmacht Droge. Rastatt: Moewig.

Doran, Jamie (Buch und Regie) (2003). »'Das ist ein schmutziges Geheimnis ...<US-Kampfpiloten unter Drogen. « [NDR-Fernsehreportage am 25.6.2003]. In: http://www.ndrtv.de/doku/20030625_2.html (Stand v. 24.1.2004).

Drug Policy Alliance (2003a). »Dance, Music and Entertainment. In: http://www. lindesmith.org/communities/raveact (Stand v. 5.10.2003).

Drug Policy Alliance (2003b). "Legislative History of the RAVE Act. « In: http://www.lindesmith.org/communities/raveact/legislative/index.cfm (Stand v. 4.3.2003).

Drug Policy Alliance (2003c). »Terrorism.« In: http://www.lindesmith.org/global/ terrorism/index.cfm (Stand v. 6.10.2003).

Giulianotti, Richard (1997). "Drugs and the Media in the Era of Postmodernity: An Archaeological Analysis. «In: Media, Culture \& Society 19, H. 3, S. 413.

Herer, Jack (1993). Die Wiederentdeckung der Nutzpflanze Hanf. Übs. v. M. Bröckers. Frankfurt/M.: Zweitausendeins.

Hutson, Scott R. (2000). "The Rave: Spiritual Healing in Modern Western Subcultures. « In: Anthropological Quarterly 73, H. 1, S. 35-49.

Jonnes, Jill (1999). Hep-Cats, Narcs and Pipe Dreams. Baltimore: John Hopkins University Press.

Kopel, Dave (2003). »Feel like Dancing? Beware of Tom Daschle.«In: http://www. nationalreview.com/kopel/kopel013003.asp (Stand v. 10.10.2003).

Kreye, Andrian (2001). »Fight for your right to party.«In: Süddeutsche Zeitung, 2. November.

Kupfer, Alexander (1996). Göttliche Gifte. Kleine Kulturgeschichte des Rausches seit dem Garten Eden. Stuttgart, Weimar: J.B. Metzler.

Leary, Timothy (1997). Denn sie wußten was sie tun. Übs. v. S. G. Seiler. München: Heyne (3. Aufl.).

Leonard, Neil (1962). Jazz and the White Americans: The Acceptance of a New Art Form. Chicago: University of Chicago Press.

McColl, William D. (2003). "Circuit Breaker.«In: Circuit Noize 36, Summer, S. 7678.

Musto, David F. (1997). "Busted - Americas War on Marihuana.« [Interview]. In: http://www.pbs.org/wgbh/pages/frontline/shows/dope/interviews/musto.html (Stand v. 3.3.2003).

Musto, David F. (1999). The American Disease: Origins of Narcotic Control. New York: Oxford University Press (3. Aufl.).

Pollack, Neal (2003). »The Right to Party.« In: http://www.brooklynrail.org/ express/june03/righttoparty.html (Stand v. 26.4.2004). 
Rätsch, Christian (Hg.) (1992). Das Tor zu inneren Räumen. Südergellsen: Verlag Bruno Martin.

Rosenbaum, Marsha (2002). "Ecstasy: America's New >Reefer Madness‘. «In: Journal of Psychoactive Drugs 34, H. 2, S. 137-142.

Rouget, Gilbert (1985). Music and Trance. A Theory of the Relations between Music and Possession. Chicago: University Press.

Shapiro, Harry (1989). Drugs \& Rock'n'Roll. Rauschgift und Popmusik. Übs. v. Peter Hiess. Wien: Hannibal Verlag.

Sloman, Larry (1998). Reefer Madness: The History of Marijuana in America. New York: St. Martin's Griffin.

Sokoni, Opio Lumumba (2004). „Congress Trying to Ban Hip Hop Shows. Prosecutors may be Raving about Jailing Hip Hoppers very soon.« In: http://www.daveyd. com/articleraveact.html (Stand v. 17.1.2004).

Sullum, Jacob (2002). »Sex, Drugs \& Techno Music. «In: http://www.mapinc.org/ newsnmdw/v01/n2069/a05.html?111 (Stand v. 1.10.2003).

Sullum, Jacob (2003). »The Chill is on - Fighting Raves, Squelching Speech.« In: http://www.reason.com/sullum/071803.shtml (Stand v. 10.8.2003).

Weil, Andrew (1998). The Natural Mind. Boston: Houghton Mifflin (3. Aufl.).

Zinberg, Norman Earl (1984). Drug, Set, and Setting: The Basis for Controlled Intoxicant Use. New Haven: Yale University Press.

\title{
Zitierte Gesetze und Gesetzesvorlagen
}

\author{
(siehe http://thomas.loc.gov.)
}

CLEAN-UP Act, 108th US Congress, 1st Sess., H.R. 834 IH Congr. Rec. H703 (2003).

Ecstasy Awareness Act, U.S. Congress, House of Representatives, 1st Sess., H.R. 2962 IH Congr. Rec. H7784 (2003).

Justice Enhancement and Domestic Security Act of 2003, US Senate, S. 22 IS Congr. Rec. S. 35 (2003).

PROTECT Act, S. 151 Sec. 608: Illicit Drug Anti-Proliferation Act (2003).

RAVE Act, 107th US Congress, 2 Sess., S. 2633 IS Congr. Rec. S. 5705-5707 (2002).

\begin{abstract}
Since 9/11 strategies of homeland security have resulted in a social climate violating civil rights. This article discusses the historical background and current development of the RAVE Act and related acts in the US, which aim to stigmatise live music and dance music events because of an alleged drug use on such events. Parental fears for their children are being used to ban parts of youth culture and its environment. Possible connections between drug use, i.e. MDMA and other social pharmacological habituations of club culture since the beginnings of last century are constructed as a stigma sponsored by moral entrepreneurs.
\end{abstract}

